\title{
A density-based material balance equation for the analysis of liquid-rich natural gas systems
}

\author{
Miao Zhang $^{1} \cdot$ Luis F. Ayala ${ }^{1}$
}

Received: 9 October 2015 / Accepted: 13 December 2015/Published online: 1 February 2016

(C) The Author(s) 2016. This article is published with open access at Springerlink.com

\begin{abstract}
This study analytically cross examines the consistency among available zero-dimensional material balance equations (MBEs) for liquid-rich gas equations and derive a new simple yet rigorous MBE starting from governing equations applicable to these systems. We propose a new zerodimensional (tank) material balance equation directly applicable to the analysis of liquid-rich (wet and retrograde) gas reservoirs expressed as a function of an equivalent gas molar density, as well as investigate and critically compare its predictions against other zero-dimensional (tank) models proposed in the past for gas reservoir cases with different amounts of condensate content (lean, intermediate and rich). All models are employed to predict reservoir performance given reservoir original-fluids-in-place and compared against benchmark examples created by numerical simulation. Actual field examples are also analyzed using existing and proposed models to test their ability to provide reliable reserve estimations using straight-line methods. The proposed densitybased equation is proven to be straightforward to implement since it is written in terms of density, which allows it be directly expressed as an extension of the dry gas MBE, while not requiring the implementation of two-phase $Z$-factors.
\end{abstract}

Keywords Liquid-rich $\cdot$ Natural gas reservoirs $\cdot$ Material balance equation

\section{List of symbols}

$B_{L} \quad$ Reservoir hydrocarbon liquid phase formation volume factor, RB/STB

Luis F. Ayala

lfay@psu.edu

1 The Pennsylvania State University, University Park, USA
$B_{V} \quad$ Reservoir vapor phase formation volume factor, $\mathrm{RB} / \mathrm{SCF}$

$G_{f g, i} \quad$ Initial surface gas in reservoir vapor phase, SCF

$G_{p} \quad$ Cumulative gas production, SCF

$G_{p T} \quad$ Total cumulative gas production, including surface gas and gaseous equivalent of produced condensates, SCF

$G_{i} \quad$ Initial gas-in-place, SCF

$G_{T} \quad$ Total initial gas-in-place, including surface gas and gaseous equivalent of produced condensates, SCF

$N_{i} \quad$ Initial condensate-in-place, STB

$N_{p} \quad$ Cumulative oil production, STB

$N_{f o, i} \quad$ Initial surface oil in reservoir liquid phase, STB

$n_{i} \quad$ Total initial hydrocarbon in place, lbmol

$n_{p} \quad$ Total cumulative hydrocarbon production, lbmol

$n_{g} \quad$ Total moles of surface gas component at reservoir conditions, lbmol

$n_{g p} \quad$ Cumulative produced moles of surface gas component, lbmol

$n_{o p} \quad$ Cumulative produced moles of surface oil component, lbmol

$n_{g}^{V} \quad$ Moles of surface gas component in reservoir vapor phase, lbmol

$n_{g}^{L} \quad$ Moles of surface gas component in reservoir liquid phase, lbmol

$n_{o}^{V} \quad$ Moles of surface oil component in reservoir vapor phase, lbmol

$n_{o}^{L} \quad$ Moles of surface oil component in reservoir liquid phase, lbmol

$\Phi \quad$ Porosity

$\rho \quad$ Molar density, $\mathrm{lbmol} / \mathrm{ft}^{3}$

$\rho_{V} \quad$ Molar density of reservoir vapor phase, $1 \mathrm{bmol} / \mathrm{ft}^{3}$

$\rho_{L} \quad$ Molar density of reservoir liquid hydrocarbon phase, lbmol/RB

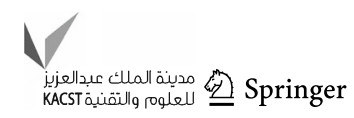


$\rho_{g s c} \quad$ Molar density of surface gas at standard condition, lbmol/SCF

$\rho_{\text {ost }} \quad$ Molar density of surface oil at stock-tank condition, lbmol/STB

$\rho_{g}^{*} \quad$ Equivalent gas molar density, lbmol/RB

$\bar{\rho}_{g}^{*} \quad$ Equivalent gas molar density at average reservoir condition, lbmol/RB

$p \quad$ Pressure, psia

$p_{\text {dew }} \quad$ Dewpoint pressure, psia

$p_{i} \quad$ Initial pressure, $\mathrm{psia}$

$t \quad$ Time, day

$T \quad$ Temperature, $\mathrm{F}$

$k \quad$ Permeability, md

$h \quad$ Thickness, $\mathrm{ft}$

$r_{e} \quad$ Reservoir outer boundary, $\mathrm{ft}$

$r_{w} \quad$ Wellbore radius, $\mathrm{ft}$

$R \quad$ Universal constant

$R_{S} \quad$ Solution gas-oil-ratio, SCF/STB

$R_{p s} \quad$ Cumulative gas-oil-ratio, $\mathrm{SCF} / \mathrm{STB}$

$R_{v} \quad$ Volatilized oil-gas-ratio, STB/SCF

$f_{n L} \quad$ Liquid phase molar fraction at reservoir conditions, fraction

$f_{n V} \quad$ Vapor phase molar fraction at reservoir conditions, fraction

$S_{V} \quad$ Vapor phase saturation, $\%$

$S_{L} \quad$ Liquid phase saturation, $\%$

$q_{g s c} \quad$ Gas production rate at standard condition, SCF/D

$q_{\text {osc }} \quad$ Oil production rate at standard condition, STB/D

$q_{V} \quad$ Vapor production rate at reservoir condition, $\mathrm{ft}^{3} / \mathrm{D}$

$q_{L} \quad$ Liquid production rate at reservoir condition, $\mathrm{RB} / \mathrm{D}$

$R V \quad$ Relative liquid dropout volume, fraction

v Velocity, $\mathrm{ft} / \mathrm{s}$

$\mathbf{v}_{\mathbf{V}} \quad$ Velocity of reservoir vapor phase, $\mathrm{ft} / \mathrm{s}$

$\mathbf{v}_{\mathbf{L}} \quad$ Velocity of reservoir liquid phase, $\mathrm{ft} / \mathrm{s}$

$V_{b} \quad$ Reservoir bulk volume, $\mathrm{ft}^{3}$

$V_{p} \quad$ Reservoir pore volume, $\mathrm{ft}^{3}$

$V_{V} \quad$ Reservoir vapor volume, $\mathrm{ft}^{3}$

$V_{L} \quad$ Reservoir liquid volume, $\mathrm{ft}^{3}$

$Z_{\mathrm{tp}} \quad$ Two-phase compressibility factor

$Z_{\text {tp,CCE }}$ Two-phase compressibility factor from CCE experimental data

$Z_{\mathrm{tp}, \mathrm{CVD}}$ Two-phase compressibility factor from CVD experimental data

\section{Introduction}

Reliable reserve estimation is a key component of economic evaluation and decision-making in reservoir development. Zero-dimensional material balance equations (MBEs) are widely deployed during these estimations to perform original fluid in place and reservoir performance predictions. The basic assumption for the zero-dimensional model is that the reservoir system can be depicted as a sealed tank where reservoir changes upon depletion can be represented as a function of volumetrically averaged average reservoir conditions. The first general material balance equation was developed by Schilthuis (1936) through equaling underground fluid withdrawal with total fluid expansion. Schilthuis's equation was limited to the analysis of black oil and dry gas reservoirs.

In this work, zero-dimensional models (tank model) for liquid-rich gases or gas-condensate reservoirs are investigated. Walsh et al. (1994a, b) derived the generalized material balance equation (GMBE) applicable to the full range of conventional reservoir fluid types. When rock and water expansion is neglected, the GMBE for an initially undersaturated gas-condensate reservoir fluid is written as follows:

$$
\begin{aligned}
& N_{p}\left(B_{L}-B_{V} R_{s}\right)+G_{p}\left(B_{V}-B_{L} R_{v}\right) \\
& \quad=G_{i}\left[R_{v i}\left(B_{L}-B_{V} R_{s}\right)-B_{V i}\left(1-R_{s} R_{v}\right)+\left(B_{V}-B_{L} R_{v}\right)\right]
\end{aligned}
$$

The derivation from Walsh et al. (1994a)'s form of GMBE to Eq. 1 is provided in Appendix 1. In all zerodimensional MBEs, such as the GMBE presented in Eq. 1, reservoir fluids are characterized using black-oil fluid formulations. In these formulations, only two pseudocomponents are 'allowed' to be present in the reservoir phases-i.e., surface gas and surface oil. It is further assumed that compositional and volumetric effects can be captured through the concepts of $R_{s}$ (solution gas-to-oil ratio), $R_{v}$ (volatilized oil-to-gas ratio), reservoir oil phase formation volume factor $\left(B_{L}\right)$ and reservoir gas phase formation volume factor $\left(B_{V}\right)$-all sole functions of pressure. Walsh et al. (1994a) showed that the GMBE in Eq. 1 may be collapsed to the well-known $p / Z$ vs. cumulative gas production $\left(G_{p}\right)$ relationship applicable to volumetric dry-gas reservoirs. For gas-condensate reservoir analysis, however, Eq. 1 may not be presented in terms of $p / Z$ vs. $G_{p}$ straight line plots because it is not pressure or $p /$ $Z$ explicit (Walsh et al. 1994a). Therefore, a single-variable Newton-Raphson procedure would need to be employed to iteratively calculate average pressure for a given resource $\left(G_{i}\right)$.

To maintain the analogy with $p / Z$ vs. $G_{p}$ MBEs for drygas systems, $p / Z_{\text {tp }}$ plots versus cumulative production plots have been proposed for the analysis of volumetric liquidrich gas systems. These models embrace the concept of 'two-phase' $Z$-factors $\left(Z_{\mathrm{tp}}\right)$ that replace customary singlephase gas $Z$-factors in dry-gas MBEs. Hagoort (1988) 
initially proposed a zero-dimensional material balance equation for volumetric gas condensate reservoirs analysis in terms of the concept of a "two-phase" Z-factor, written as follows:

$\frac{\bar{p}}{\bar{Z}_{\mathrm{tp}}}=\frac{p_{i}}{Z_{\mathrm{tp}, i}}\left(1-\frac{n_{p}}{n_{i}}\right)$

where $n_{p}$ is total produced hydrocarbon moles and $n_{i}$ is total initial hydrocarbon moles in place written in terms of total moles using the black-oil fluid formulation-i.e., in terms of surface gas (g) and surface oil (o) components where $n_{p}=n_{g p}+n_{o p}$ and $n_{i}=n_{g i}+n_{o i}$. Hagoort (1988) rewrote Eq. 2 in terms of cumulative surface gas production $\left(G_{p}\right)$ and surface oil production $\left(N_{p}\right)$ by relating total molar quantities to cumulative production and proposed:

$\frac{\bar{p}}{\bar{Z}_{\mathrm{tp}}}=\frac{p_{i}}{Z_{\mathrm{tp}, i}}\left[1-\frac{G_{p}\left(1+R_{\mathrm{MLG}_{p}}\right)}{G_{i}\left(1+R_{\mathrm{MLG}_{i}}\right)}\right]$

where $R_{\mathrm{MLG}_{i}}$ and $R_{\mathrm{MLG}_{p}}$ are Hagoort's initial and cumulative condensate/gas molar ratios defined as:

$R_{\mathrm{MLG}_{i}}=\frac{n_{o i}}{n_{g i}}=\frac{R_{v i} G_{i} \rho_{\text {ost }}}{G_{i} \rho_{g s c}}=\frac{\rho_{\text {ost }}}{\rho_{\text {gsc }}} \cdot R_{v i}$

$R_{\mathrm{MLG}_{p}}=\frac{n_{o p}}{n_{g p}}=\frac{N_{p} \rho_{\text {ost }}}{G_{p} \rho_{g s c}}=\frac{\rho_{\text {ost }}}{\rho_{g s c}} \cdot \frac{N_{p}}{G_{p}}$

where the gas molar density at standard conditions $\left(\rho_{g s c}\right.$, $\mathrm{lbmol} / \mathrm{SCF}$ ) is a constant equal to $0.002634 \mathrm{lbmol} / \mathrm{ft}^{3}$ for ideal gases and the molar density of the stock tank $\left(\rho_{\text {ost }}\right.$, lbmol/STB) is estimated directly from stock tank oil API (Walsh and Lake 2003). In black-oil fluid formulations, produced hydrocarbon phases at the surface are 'pure' single-component fluids, thus these densities correspond to those of the 'pure' fluids containing $100 \%$ of surface gas component and surface oil components, respectively. An identical expression to Eq. 3 is obtained if, instead of using Hagoort's $R_{\mathrm{MLG}_{i}}$ and $R_{\mathrm{MLG}_{p}}$ definitions, one directly substitutes $\quad n_{p}=\int_{0}^{t}\left(q_{g s c} \rho_{g s c}+q_{o s c} \rho_{\text {ost }}\right) \mathrm{d} t=G_{p} \rho_{g s c}+$ $N_{p} \rho_{\text {ost }}$ and $n_{i}=G_{i}\left(\rho_{g s c}+\rho_{\text {ost }} R_{v i}\right)$ into Eq. 2. The terms $G_{p}\left(1+R_{\mathrm{MLG}_{p}}\right)$ and $G_{i}\left(1+R_{\mathrm{MLG}_{i}}\right)$ in Eq. 3 are also referred as 'total cumulative gas production' $\left(G_{p T}\right)$ and 'total initial gas in place' $\left(G_{T}\right)$, respectively, which are variables that lump the surface gas and the gaseous equivalent of produced condensates together. This leads to the following more compact form of this MBE:

$\frac{\bar{p}}{\bar{Z}_{\mathrm{tp}}}=\frac{p_{i}}{Z_{\mathrm{tp}, i}}\left(1-\frac{G_{p T}}{G_{T}}\right)$

which has been also presented by Lee and Wattenbarger (1996) as the applicable MBE for volumetric gas- condensate reservoirs. When coupled with production history, deployment of Eqs. 2, 3, or Eq. 6 requires a priori knowledge of 'two-phase' $Z$-factors $\left(Z_{\mathrm{tp}}\right)$ and volatilized oil-gas-ratios $\left(R_{v}\right)$ data as a function of pressure. These input variables are routinely estimated from constant volume depletion (CVD) PVT laboratory tests or via a properly tuned equation of state (EOS). From here, it follows that a plot of $\frac{\bar{p}}{\bar{Z}_{\mathrm{tp} . \mathrm{CVD}}}$ against total cumulative gas production $\left(G_{p T}\right)$ yields total initial gas in place $\left(G_{T}\right)$ at the $x$-axis intercept resulting from the best-fit straight-line extrapolation. This methodology assumes that $Z_{\mathrm{tp}, \mathrm{CVD}}$ obtained from the laboratory test is applicable to the study of interest, which tends to be a good approximation when reservoir condensate remains immobile-a basic premise honored by experimental CVD tests. When condensate is highly mobile, this approximation may no longer hold. This is further examined in subsequent sections in this manuscript.

Vo et al. (1990) showed that "two-phase" $Z$-factors $\left(Z_{\mathrm{tp}}\right)$ are molar-weighted averages of the $Z$-factors of the reservoir gas and oil phases, i.e., $Z_{\mathrm{tp}}=f_{n L} Z_{L}+f_{n V} Z_{V}$. Twophase $Z$-factors, as the $Z$-factors of the entire hydrocarbon mixture inside a PVT cell, are straightforwardly calculated for any two-phase system on the sole basis of pressure, temperature, volume, and total hydrocarbon moles data obtained from either CVD tests-where gas is continuously produced/removed from the PVT cell-or from constant composition expansion (CCE) tests-where no gas production is realized from the PVT cell. Vo et al. (1990) further illustrated the approximate validity of the following empirical simplification of the $Z_{\mathrm{tp}}$ MBE:

$\frac{\bar{p}}{\bar{Z}_{\mathrm{tp}, \mathrm{CCE}}}=\frac{p_{i}}{Z_{\mathrm{tp}, i}}\left(1-\frac{G_{p}}{G_{i}}\right)$

Equation 7 is written in terms of surface gas cumulative production $\left(G_{p}\right)$ rather than total produced surface gas moles $\left(n_{p}\right)$ or total cumulative gas production $\left(G_{p T}\right)$ as Eqs. 2, 3, or Eq. 6 require. To accomplish this, the authors indicate that $Z_{\text {tp }}$ values should be collected from $C C E$ tests-and not from CVD tests as with the previous models. The applicability of Eq. 7 for gas-condensate analysis was verified in practice, with satisfactory results, not only by Vo et al. (1990) using numerical cases but also later with field examples (e.g., Zeidouni et al. 2006). As with any approximation, whether Eq. 7 remains generally applicable for all or most cases of interest is debatable. The implications of using of $Z_{\mathrm{tp}, \mathrm{CVD}}$ instead of the recommended $Z_{\mathrm{tp}, \mathrm{CCE}}$ in Eq. 7 are not always clear. This is further examined in subsequent sections in this article.

A recent attempt at deploying two-phase $Z$-factor in liquid-rich MBE analysis has been presented by Sureshjani 
et al. (2014). They proposed the use of a modified twophase $Z$-factor $\left(Z_{\mathrm{tp}}^{*}\right)$ along with the following gascondensate MBE in terms of cumulative gas production $\left(G_{p}\right)$ derived from a statement of surface gas conservation:

$\frac{\bar{p}}{\bar{Z}_{\mathrm{tp}}^{*}}=\frac{p_{i}}{Z_{\mathrm{tp}, i}^{*}}\left(1-\frac{G_{p}}{G_{i}}\right)$

where $Z_{\mathrm{tp}}^{*}$, the modified two-phase $Z$-factor, is defined by the authors as:

$Z_{\mathrm{tp}}^{*}=Z_{t p} \frac{T_{s c} R}{p_{s c}}\left[\frac{\left(\frac{1}{R V}-1\right) \rho_{V}+\rho_{L}}{\left(\frac{1}{R V}-1\right) \frac{1}{B_{V}}+\frac{R_{s}}{B_{L}}}\right]$

where $Z_{\mathrm{tp}}$ is the CVD two-phase Z-factor, $R V$ is the relative liquid-dropout volume from the CVD test, and $\rho_{V}$ and $\rho_{L}$ are the molar density of reservoir gas and oil phases, respectively. As described, Sureshjani et al. (2014) suggest that all parameters required for the calculation of $Z_{\mathrm{tp}}^{*}$ in Eq. 9 should be obtained from CVD data.

This study investigates and compares predictions from these currently available zero-dimensional models for liquid-rich gas (gas-condensate) reservoirs, including the generalized material balance equation (GMBE) and all two-phase Z-factor MBEs described above, along with the presentation of a new model that uses the concept of equivalent gas molar density and does not implement two-phase Z-factors. Assumptions behind each of these models are discussed. They are also deployed to predict reservoir pressure depletion given known values of reservoir original-gas-in-place and for reserve estimations. Model performances are compared against each other and against reported data from finely gridded reservoir compositional simulation and actual field examples.

\section{A density-based liquid-rich MBE}

Molar conservation of surface gas component can be expressed in terms of total surface-gas hydrocarbon moles remaining in situ at any point in time $\left(n_{g}\right)$ as follows:

$n_{g}=n_{g i}-n_{g p}$

where $n_{g}=n_{g}^{V}+n_{g}^{L}$. By defining an equivalent gas molar density, $\rho_{g}^{*}$, as total moles of surface gas present at reservoir conditions per unit reservoir pore volume, we can write its volumetric average $\bar{\rho}_{g}^{*}$ as:

$\bar{\rho}_{g}^{*}=\frac{\iiint \rho_{g}^{*} \mathrm{~d} V_{p}}{V_{p}}=\frac{n_{g}}{V_{p}}=n_{g} \cdot \frac{\rho_{g, i}^{*}}{n_{g i}}$ where $\bar{\rho}_{g}^{*}$ represents the equivalent gas molar density evaluated at the average reservoir pressure condition. Substitution of Eq. 10 into Eq. 11 leads to:

$\frac{\bar{\rho}_{g}^{*}}{\rho_{g, i}^{*}}=1-\frac{n_{g p}}{n_{g i}}$

It is readily noted that an "equivalent" gas molar density $\left(\rho_{g}^{*}\right)$ is not the molar density of reservoir gas phase, as it only represents the amount of moles of surface gas component remaining in situ per unit reservoir pore volume as prescribed by Eq. 11. $\rho_{g}^{*}$ rather represents the density that the in situ surface gas $\left(n_{g}\right.$, found in the free gas and solution gas) would have if it were single-phase at the given reservoir condition. Using its definition in Eq. 11, this equivalent gas molar density $\rho_{g}^{*}$ can also be calculated in terms of standard PVT black-oil properties and saturation data as follows:

$\bar{\rho}_{g}^{*}=\frac{n_{g}^{V}+n_{g}^{L}}{V_{p}}=\frac{\rho_{g s c} \frac{V_{V}}{B_{V}}+\rho_{g s c} \frac{V_{L}}{B_{L}} R_{s}}{V_{p}}=\rho_{g s c} \cdot\left(\frac{S_{V}}{B_{V}}+\frac{S_{L}}{B_{L}} R_{s}\right)$

where $\rho_{g s c}$ is the surface gas molar density at standard conditions, and $V_{V}$ and $V_{L}$ are the vapor phase and liquid phase volumes at the given reservoir condition, respectively. Given the direct proportionality between moles and surface gas quantities, the proposed liquid-rich MBE expressed in terms of equivalent density is derived from Eq. 12 as shown:

$\bar{\rho}_{g}^{*}=\rho_{g, i}^{*} \cdot\left(1-\frac{G_{p}}{G_{i}}\right)$

Equation 14 shows that liquid-rich MBEs can indeed be rigorously written in term of surface gas production $G_{p}$ and equivalent gas molar density, as per the requirements of surface gas component molar conservation. Equation 14 provides a rigorous black-oil model in term of $G_{p}$ (surface gas production) in direct analogy with the traditional $p / Z$ (or density) vs. $G_{p}$ dry-gas models. Indeed, a best-fit straightline in a $\bar{\rho}_{g}^{*}$ vs. $G_{p}$ plot will intercept $x$-axis at $G_{i}$, as shown in subsequent sections. Also, for reservoir conditions higher than dewpoint, Eq. 14 readily collapses to the density-based dry gas MBE $: \bar{\rho}=\rho_{i}\left(1-\frac{G_{p}}{G_{i}}\right)$. It is noted that original gas in place $G_{i}$ can be expressed in terms of $\rho_{g i}^{*}$ as:

$G_{i}=\frac{\rho_{g, i}^{*} V_{p}}{\rho_{g s c}}=\frac{\rho_{g, i}^{*}}{\rho_{g s c}} A h \phi$

Since $B_{L}, B_{V}$ and $R_{s}$ are pressure-dependent black-oil PVT properties, Eq. 13 requires a pressure-saturation relationship for the calculation of $\rho_{g}^{*}$. We show that the CVD liquid dropout volume $S_{L, \mathrm{CVD}}$ vs. pressure profile is a 
good approximations for these purposes. As a result, Eq. 14 can be straightforwardly applied without further manipulation of input data or iterations given that all required input parameters $\left(B_{L}, B_{V}\right.$ and $R_{S}$ and $\left.S_{L}\right)$ are directly obtained from standard CVD tests. In this way, an evenly spaced table of $p$ vs. $\rho_{g}^{*}$ can be straightforwardly made prior to analysis. Average pressures may be then explicitly interpolated from this table after $\bar{\rho}_{g}^{*}$ values are obtained using Eq. 14. In addition, being able to connect liquid-rich MBE calculations directly to density estimations readily enables the extension of density-based decline analysis methods (see Ayala and Ye 2013; Zhang and Ayala 2014) to liquid-rich gas condensate wells.

\section{Liquid-rich MBEs: classical vs. numerical models}

Zero-dimensional material balance equations (MBEs) describe reservoir dynamics in terms of a volume-averaged condition that neglects (pressure and saturation) gradients (i.e., "tank" model). In numerical simulation, fully dimensional flow models are solved through discretized versions of the applicable partial differential equations. Both approaches are based on mass conservation, thus classical material balance equations (MBEs) must be able to be directly derived from their partial differential counterparts used in numerical simulation. Partial differential equations for multiphase flow in porous media can be written in terms of compositional or black-oil fluid formulations. In the compositional formulation, for example, the conservation principle is applied to each individual component. In this case, the continuity equation for the flow of component (' $i$ ') becomes:

$-\nabla \cdot\left(\rho_{V} \mathbf{v}_{V} y_{i}+\rho_{L} \mathbf{v}_{L} x_{i}\right)=\frac{\partial}{\partial t}\left[\phi\left(\rho_{V} S_{V} y_{i}+\rho_{L} S_{L} x_{i}\right)\right]$

where $\rho_{V}$ and $\rho_{L}$ are the molar densities of reservoir vapor and liquid phase, $\mathbf{v}_{\boldsymbol{V}}$ and $\mathbf{v}_{\boldsymbol{L}}$ represent fluxes of reservoir vapor and liquid, and $y_{i}$ and $x_{i}$ are the molar fraction of component $i$ in vapor and liquid phases, respectively. In these compositional formulations, the entire system is assumed at thermodynamic equilibrium and each of hydrocarbon phases may contain many components. In black-oil formulations, only two pseudo-components are allowed in hydrocarbon phases-namely, surface gas and surface oil. Compositional and volumetric effects in blackoil models are captured through the concepts of $R_{s}$ (solution gas-to-oil ratio), $R_{v}$ (volatilized oil-to-gas ratio), reservoir oil formation volume factor $\left(B_{L}\right)$ and reservoir gas formation volume factor $\left(B_{V}\right)$. For such conditions, and for the surface gas component, Eq. 16 collapses to: $-\nabla \cdot\left(\frac{\mathbf{v}_{V}}{B_{V}}+\frac{\mathbf{v}_{L}}{B_{L}} R_{s}\right)=\frac{\partial}{\partial t}\left[\phi\left(\frac{S_{V}}{B_{V}}+R_{s} \frac{S_{L}}{B_{L}}\right)\right]$

For the surface oil component, Eq. 16 collapses to:

$-\nabla \cdot\left(\frac{\mathbf{v}_{L}}{B_{L}}+\frac{\mathbf{v}_{V}}{B_{V}} R_{v}\right)=\frac{\partial}{\partial t}\left[\phi\left(\frac{S_{L}}{B_{L}}+R_{v} \frac{S_{V}}{B_{V}}\right)\right]$

All liquid-rich classical material-balance methods are written in terms of black-oil fluid formulations and thus can be derived from either Eqs. 17 or 18, or a combination of both. As a result, zero-dimensional MBEs are bound to reproduce fully dimensional numerical data results when properly applied and for conditions consistent with their assumptions. These connections are demonstrated in the Appendices.

Appendix 1 shows that, upon integration of Eqs. 17 and 18, these PDEs collapse to the GMBE form presented in Eq. 1 as derived by Walsh et al. (1994a). Appendix 2 shows that Hagoort's $Z_{\mathrm{tp}}$ MBE (Eq. 2) is derived from an overall molar conservation statement that also follows from the combined application of the volume-integrated Eqs. 17 and 18. GMBE and Hagoort's $Z_{\text {tp }}$ MBE are both based on enforcing overall mass conservation but: (a) GMBE employs gas and oil component molar conservation statements written independently in term of black-oil properties; (b) Hagoort's $Z_{\mathrm{tp}}$ MBE considers molar conservation of total hydrocarbon (surface gas and oil moles together, undistinguished) expressed in term of total molar recovery.

As discussed previously, Vo's CCE-based $Z_{\mathrm{tp}}$ MBE (Eq. 7) is an empirical simplification with practical applications. It may actually be derived from Hagoort's Eq. 3 for any hypothetically created condition that would allow $R_{\mathrm{MLG}_{p}} \approx R_{\mathrm{MLG}_{i}}$-i.e., $\frac{N_{p}}{G_{p}} \sim R_{v i}$. Such equivalency $R_{v i}=\frac{N_{p}}{G_{p}}$ would be only valid for single-phase depleting systems (such as dry and wet gases or liquid-rich systems with minimal reservoir condensation) which remain constantcomposition at reservoir conditions throughout depletion. As a result, application of Vo's Eq. 7 indeed requires the creation of a fictitious constant-composition producing stream and $Z_{\mathrm{tp}}$ reservoir data consistent with it, which can only be obtained through a CCE test. For such hypothetical stream, $R_{\mathrm{MLG}_{p}, \mathrm{CCE}}$ would remain almost unchanged and equal to initial $R_{\mathrm{MLG}_{i}}$ throughout the expansion process. In actual systems, two-phase production leads to significant reservoir composition changes-conditions that are best captured by CVD tests. Therefore, the application of Eq. 7 with CVD-based $Z_{\text {tp }}$ data would not be valid and leads to poor predictions.

Equation 7 (Vo's CCE-based $\left.Z_{\mathrm{tp}} \quad \mathrm{MBE}\right), \quad$ Eq. 8 (Sureshijani's CVD-based $Z_{\mathrm{tp}}^{*} \mathrm{MBE}$ ), and Eq. 14 (proposed 
Table 1 Gas reservoir fluid PVT properties

\begin{tabular}{lllll}
\hline & Lean gas A & Mid gas B & Rich gas C & Rich gas D \\
\hline Initial pressure, $p_{i}$ (psia) & 3500 & 3000 & 3000 & 3500 \\
Dew point pressure, $p_{\text {dew }}($ psia) & 3275.0 & 2912.7 & 2787.7 & 3145.2 \\
Temperature, $T(\mathrm{~F})$ & 200 & 200 & 250 & 250 \\
Maximum CVD liquid dropout, $\% S_{o}$ & 4.6 & 12.5 & 22.2 & 33.2 \\
Initial volatilized-oil content $\left(R_{v i}\right.$, STB/MMSCF) & 47.3 & 82.4 & 129.8 \\
\hline
\end{tabular}

density-based MBE) exemplify available MBEs written in term of surface gas production $G_{p}$ only-i.e., without requiring cumulative oil production data, $N_{P}$-thus providing direct liquid-rich gas analogs to the dry-gas model. Vo et al. (1990)'s model only approximately enforces overall conservation of all components, and the absence of oil cumulative production $\left(N_{P}\right)$ in the equation is a consequence of the constant cumulative condensate/gas molar ratios characteristic of CCE processes. Both Sureshijani's CVD-based $Z_{\mathrm{tp}}^{*} \mathrm{MBE}$ (Eq. 8) and the proposed equivalent gas density MBE (Eq. 14) enforce species conservation (surface gas), rather than overall molar conservation, and thus predictions from these models must be consistent. The proposed formulation, however, does not require the definition of two-phase $Z$-factors and more directly connects to physical quantities (density) and principles. Notably, the equivalent gas molar density $\rho_{g}^{*}$ expression in Eq. 13 directly tracks the surface gas-component storage term $\left(\frac{S_{V}}{B_{V}}+\frac{S_{L}}{B_{L}} R_{S}\right)$ found on the right-hand-side of the surfacegas PDE (Eq. 17) and thus better bridges MBE calculations with multiphase performance applications. Well performance predictions, for example, can indeed be directly linked to $\rho_{g}^{*}$ time changes by combining the integrated version of Eq. 17 (see Eq. 19, Appendix 1) with the equivalent gas molar density $\rho_{g}^{*}$ expression in Eq. 13 .

\section{Case studies: numerical examples}

In this section, four different natural gases of varying degrees of initial volatilized condensate contents are considered, as presented in Table 1. These fluids include Lean gas A, Mid gas B, Rich gas C, and Rich gas D with initial volatized oil-to-gas ratios $\left(R_{v i}\right)$ of $47.3,82.4,129.8$ and 166.8 STB/MMSCF and maximum liquid dropout saturations of $4.6,12.5,22.5$ and $33.2 \%$, respectively, as depicted in Fig. 1. All relevant CVD and CCE versus pressure data for these fluids are presented in Appendix 3. These PVT tests and resulting data were simulated for the fluids of interest using the same Peng-Robinson equation of state (PR-EOS) employed by the compositional simu-

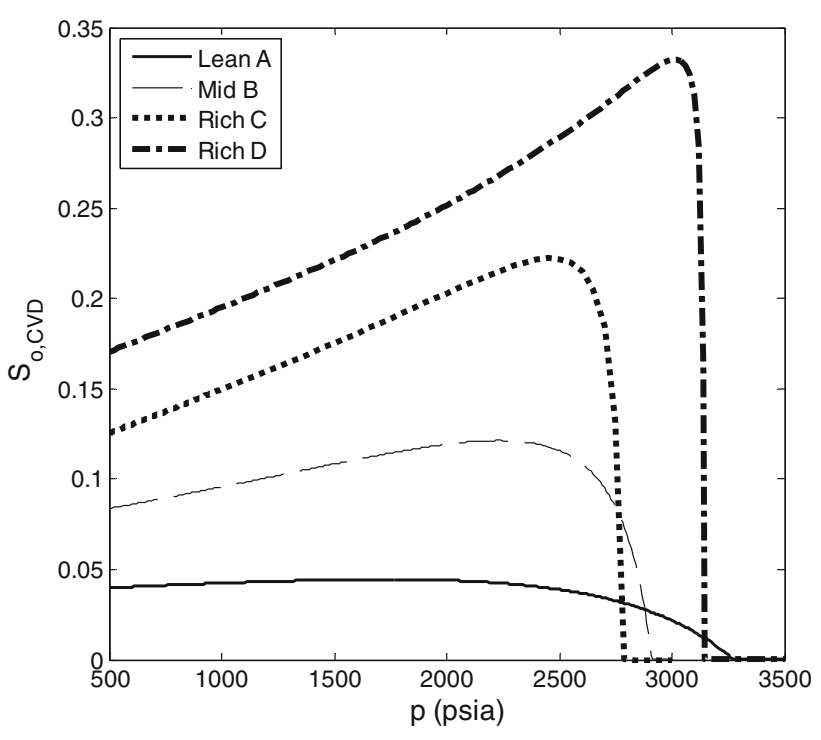

Fig. 1 CVD liquid drop-out curve of liquid-rich gases A, B, C and D

lator, to maintain consistency in performance comparisons. Table 2 displays the various combinations of depletion scenarios considered for each of these liquid-rich gas fluids, including type of production specification (constant bottomhole pressure, BHP, or constant surface gas specification) along with type of relative permeability curve inputted to the compositional numerical simulator. Three sets of relative permeability curves are considered (Set A, B, and C), as displayed in Fig. 2. $k_{r}$-curves from Set A $\left(S_{o c}=0.40\right)$ corresponds to a multiphase system where the condensate would stay mostly immobile for all fluids under consideration, given the maximum liquid saturations of Fig. 1. Condensate would mobilize when Set B of the $k_{r^{-}}$ curves $\left(S_{o c}=0.10\right)$ is used for rich C and D gases. The highest degree of condensate mobility would be attained when Set C of the $k_{r}$-curves is used. No connate water is considered.

For all case studies presented in Table 2, reservoir pressure responses were numerically generated via finely ridded compositional simulations for a 1-D radial-cylindrical system using the reservoir properties listed in 
Table 2 Numerical case studies

\begin{tabular}{llll}
\hline Case study & Fluid & Production specification & $k_{r}$-curves \\
\hline 1 & Lean gas A & Constant BHP (500 psia) & Set A \\
2 & Mid gas B & Constant $q_{\text {gsc }}(500$ MSCFD) & Set B \\
3 & Rich gas C & Constant BHP (500 psia) & Set A \\
4 & Rich gas D & Constant BHP (500 psia) & Set B \\
5 & Rich gas C & Constant $q_{\text {gsc }}(500$ MSCFD) & Set C \\
6 & Rich gas D & Constant $q_{\text {gsc }}(500$ MSCFD) & Set C \\
\hline
\end{tabular}

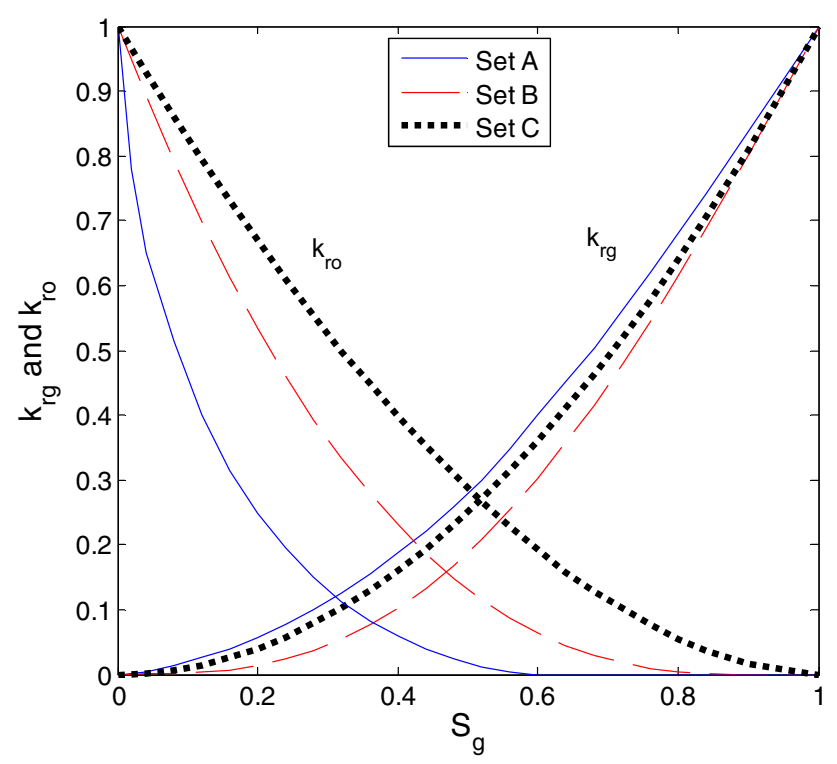

Fig. 2 Relative permeability for synthetic cases

Table 3 Reservoir properties and initial fluids in place

\begin{tabular}{ll}
\hline Absolute permeability, $k(\mathrm{md})$ & 1 \\
Porosity, $\phi$ (fraction) & 0.30 \\
Wellbore radius, $r_{w}(\mathrm{ft})$ & 0.25 \\
Reservoir thickness, $h(\mathrm{ft})$ & 50 \\
Wellbore outer boundary, $r_{e}(\mathrm{ft})$ & 500 \\
Gas-in-place, $G_{i}(\mathrm{BSCF})$ & \\
Lean gas A & 2.48 \\
Mid gas B & 2.42 \\
Rich gas C & 2.15 \\
Rich gas D & 2.28 \\
Condensate-in-place, $N_{i}(\mathrm{MMSTB})$ & \\
Lean gas A & 0.12 \\
Mid gas B & 0.20 \\
Rich gas C & 0.28 \\
Rich gas D & 0.38 \\
\hline
\end{tabular}

Table 3 and the reported initial fluids in place. These pressure responses were volumetrically averaged for direct comparison against average pressure predictions from

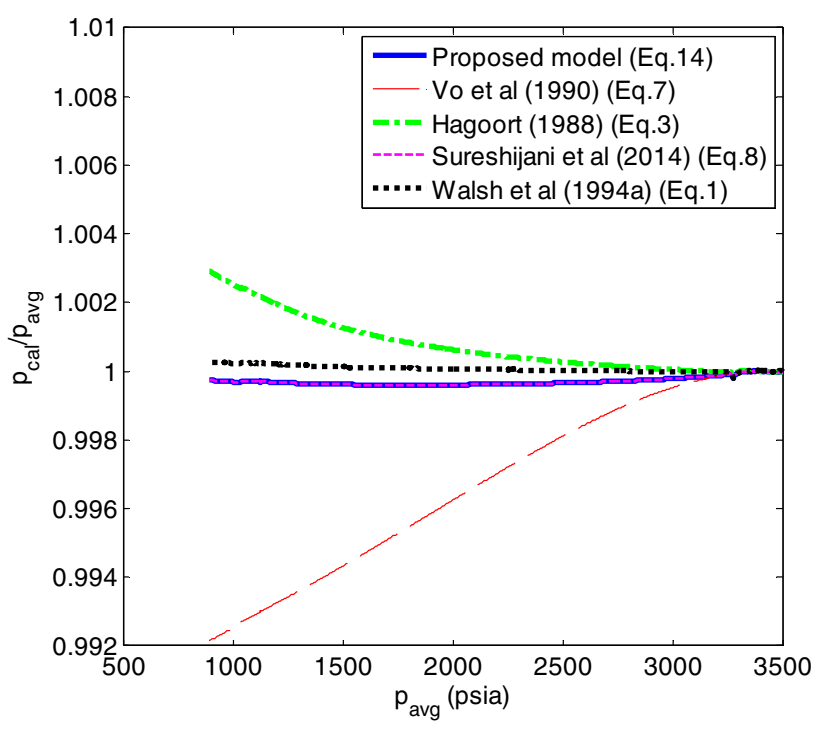

Fig. 3 Pressure prediction comparison-Case Study 1: Lean gas A Set A

available zero-dimensional liquid-rich gas MBEs discussed above: Walsh's GMBE (Eq. 1), Hagoort's $Z_{\mathrm{tp}} \mathrm{MBE}$ (Eq. 3), Vo's CCE-based $Z_{\mathrm{tp}}$ MBE (Eq. 7), Sureshijani's CVD-based $Z_{\mathrm{tp}}^{*}$ MBE (Eq. 8), and the proposed densitybased MBE (Eq. 14). Figures 3, 4, 5 and 6 compare predictions from each zero-dimensional model $\left(p_{\text {cal }}\right)$ versus actual average pressure reported by finely gridded numerical simulation $\left(p_{\text {avg }}\right)$ for Case Study 1 (Fig. 3), Case Study 2 (Fig. 4), Case Study 3 (Fig. 5) and Case Study 4 (Fig. 6). In these figures, $p_{\mathrm{cal}} / p_{\text {avg }}$ ratios are reported as a function of pressure depletion. In these figures, the best-performing zero-dimensional model should be able to remain as close as possible to the unit (1) ratio. It is observed that, for all case studies, all MBEs are able to remain within less than $4 \%$ error and are thus able to provide predictions quite close to the benchmark, though the difference becomes more noticeable as the fluid gets richer. Notably, Vo et al. (1990)'s model (Eq. 7) tends to yield the largest, yet still relatively small, errors for the first three case studies; while the proposed density-based MBE provides results quite consistent with GMBE and Sureshijani's $Z_{\mathrm{tp}}^{*}$ MBE with the advantages noted earlier. Hagoort's $Z_{\mathrm{tp}} \mathrm{MBE}$, although not 


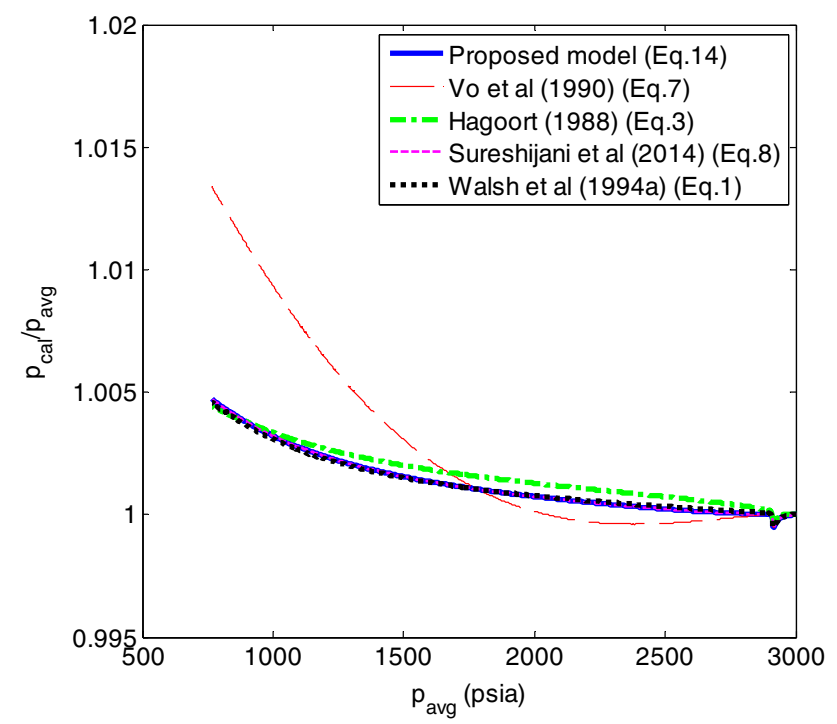

Fig. 4 Pressure prediction comparison-Case Study 2: Mid gas B, Set B

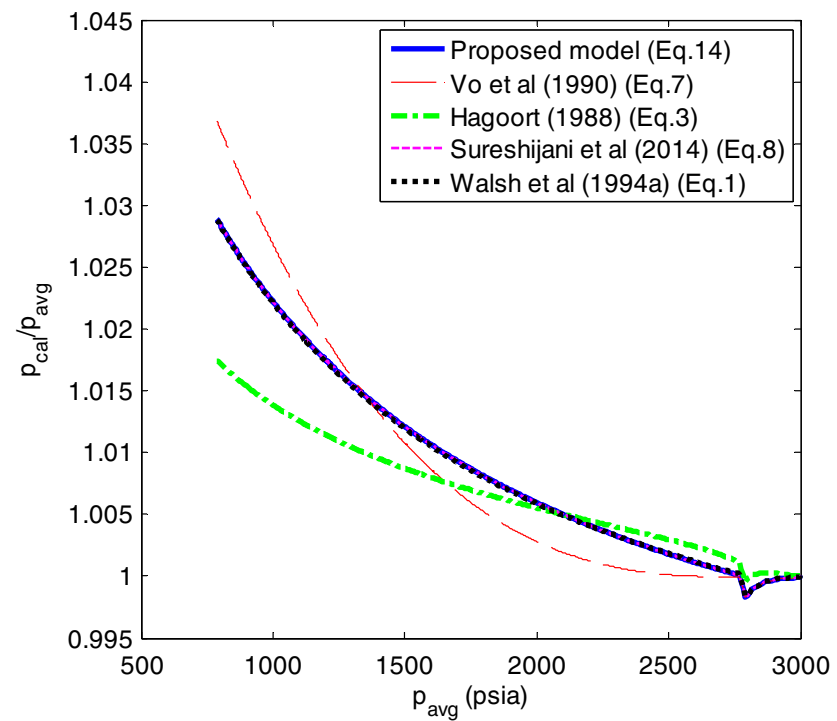

Fig. 5 Pressure prediction comparison-Case Study 3: Rich gas C, Set A

consistently the closest to the unit (1) ratio in all these figures, tends to perform slightly better as the reservoir fluid gets enriched.

Two additional figures (Figs. 7, 8) display the combination of rich gas condensate $(\mathrm{C}$ and $\mathrm{D})$ with highly mobile condensate (Set $\mathrm{C}$ ) for the examination of more extreme scenarios with significant condensate mobility (i.e., Case studies 5 and 6 in Table 2). An interesting trend emerges by directly comparing them against Figs. 5, 6 given that the only difference among them is the set of relative permeability curves being used. Hagoort's $Z_{\mathrm{tp}} \mathrm{MBE}$ (Eq. 3)

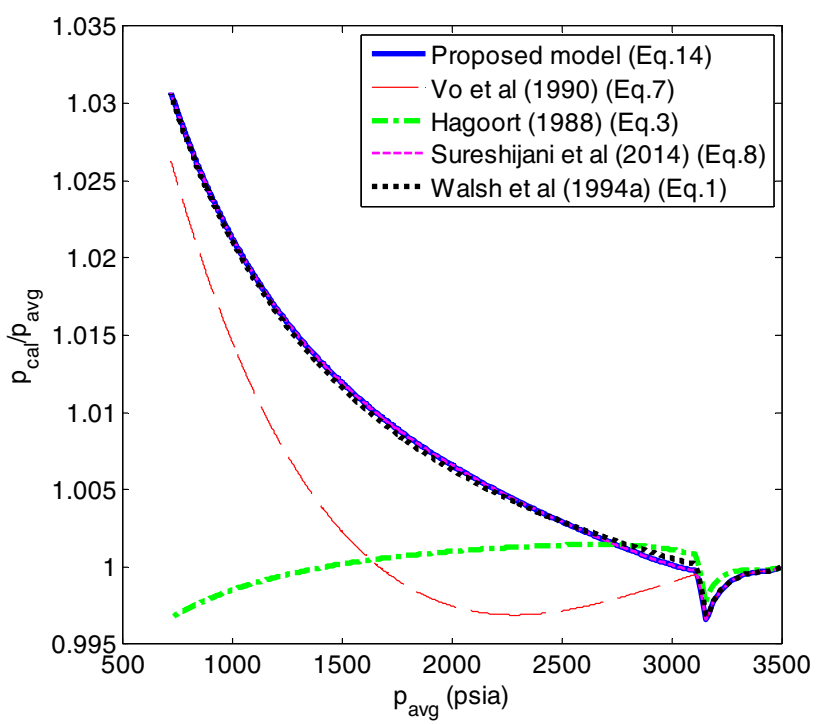

Fig. 6 Pressure prediction comparison-Case Study 4: Rich gas D, Set B

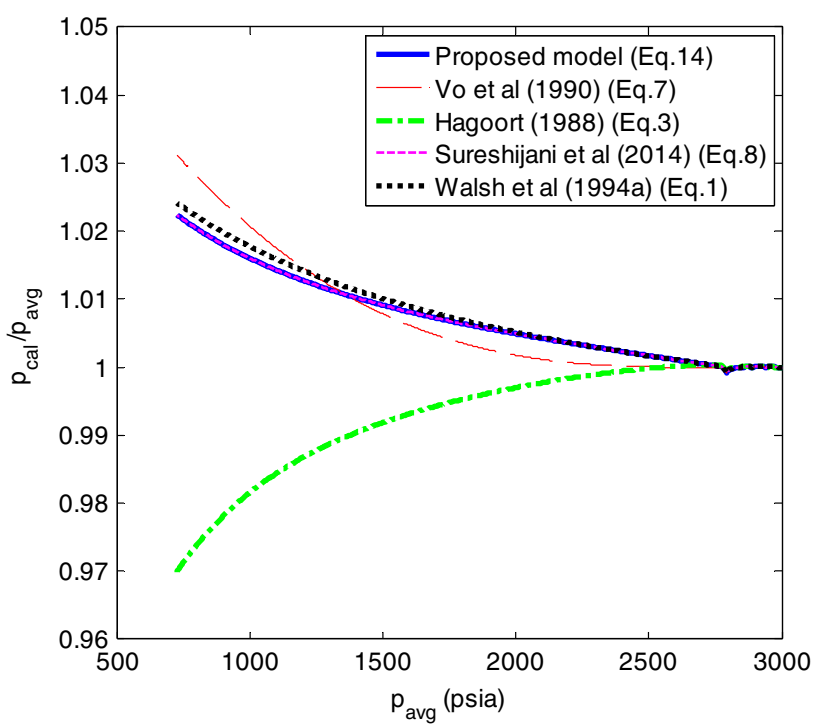

Fig. 7 Pressure prediction comparison for Case Study 5: Rich gas C, Set C

predictions worsen when condensate becomes highly mobile. This is an indication that the $\left(Z_{\mathrm{tp}}, R_{\mathrm{MLGP}}\right)$ coupling assumed by Eq. 3 at the given pressure depletion step from CVD data becomes more tenuous as condensate mobility increases. Figures 7 and 8 also show that the proposed density model using surface gas production data only, also closely followed by GMBE and Sureshijani's $Z_{\mathrm{tp}}^{*} \mathrm{MBE}$, proves to be more reliable for the analysis of these higher oil mobility cases. The effect of condensate mobility in the proposed model is introduced by pressure-saturation relationship used when evaluating $\rho_{g}^{*}$ in Eq. 13: as condensate 


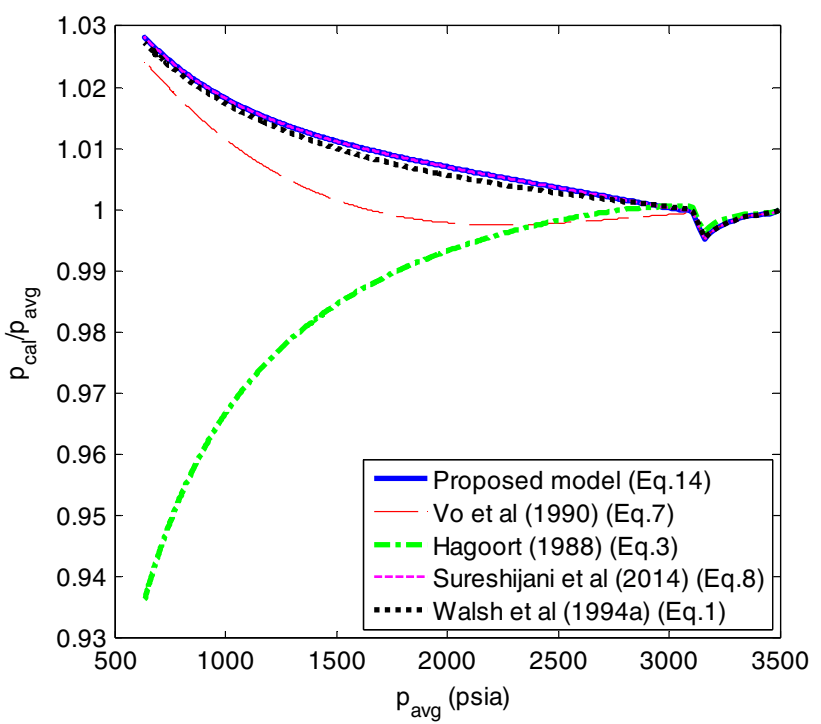

Fig. 8 Pressure prediction comparison-Case Study 6: Rich gas D, Set C

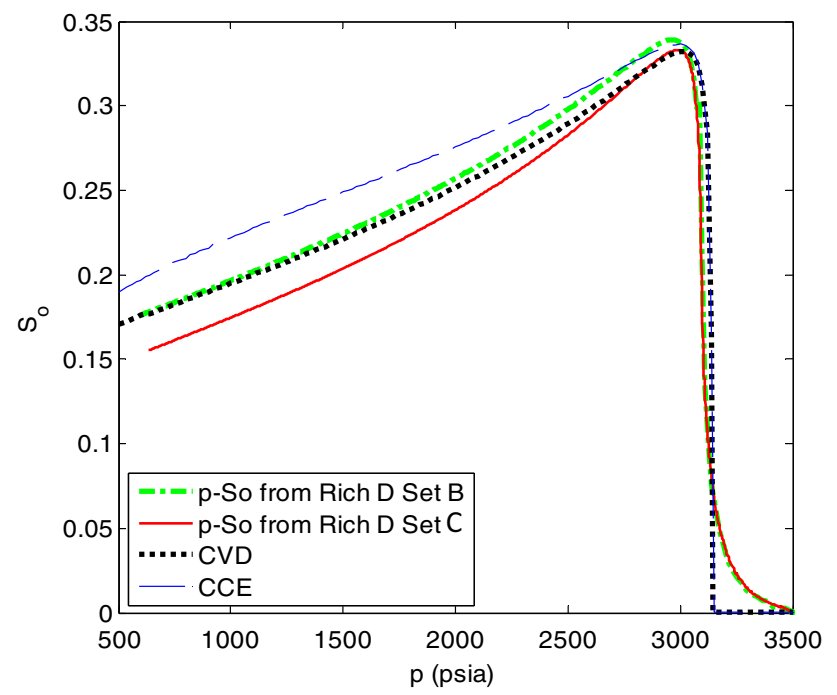

Fig. 9 Average $p$-So relationship for Rich gas D versus CVD and CCE data

mobility increases, the average pressure-saturation profile differs from the one reported by the CVD test. Figure 9 illustrates such condensate mobility effect on the pressuresaturation relationship for Rich Gas D for the cases when condensate is nearly immobile ( $k_{r}$-curves Set B) and highly mobile (Set $\mathrm{C}$ ) compared to results reported by numerical simulation. When condensate is close to immobile (Set B), actual average $S_{o}-p$ tend to more closely follow CVDreported trends (Fig. 7). As condensate mobilizes (Set C), actual average $S_{o}-p$ data deviate from CVD trends. Figures 7 and 8 also show that the proposed MBE is less

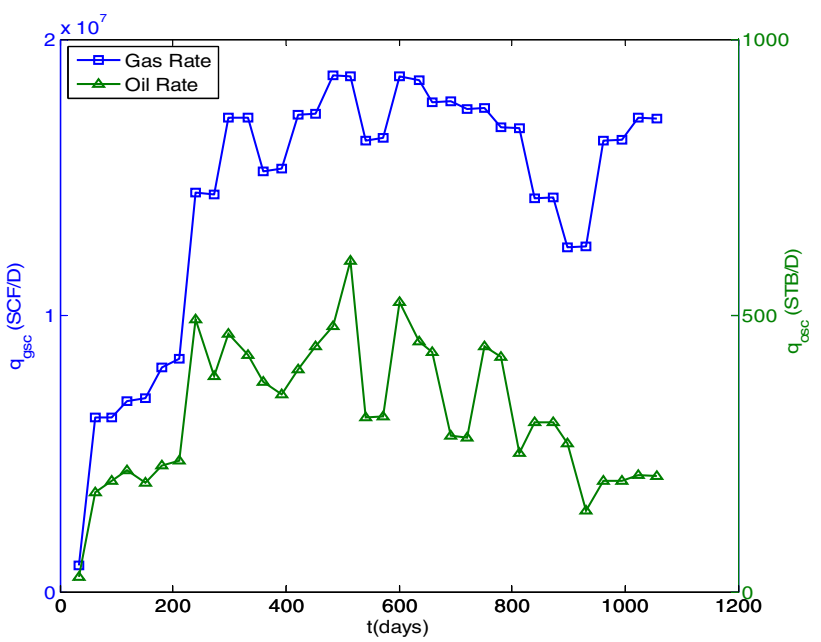

Fig. 10 Production history-Field case A

affected by this pressure-saturation disparity than the inputs used by Hagoort's Eq. 3.

\section{Case studies: field examples}

Two field examples are analyzed in this section to illustrate the applicability of proposed MBE for reserve estimations. Field Case A considers a lean gas condensate reservoir in Algeria presented and studied by Bengherbia and Tiab (2002) using compositional simulation. Starting at an initial pressure of 2683 psia, slightly higher than dewpoint pressure (2619 psia), two-phase flow occurs after a short production time. Reported production history is shown in Fig. 10 and reported original gas- and condensate-in-place are 67.1 BSCF and 1.9 MMSTB, respectively. For the purpose of zero-dimensional MBE analysis, CVD and CCE test data was generated using the Peng Robinson EOS for the reported fluid composition. Results of the analysis using the proposed density-based MBE (Eq. 14) is shown in Fig. 11 as $G_{i}=65.1$ BSCF. Original condensate-inplace estimation corresponds to $G_{i} \times R_{v i}=1.7$ MMSTB, both estimations showing good agreement with reported values. For comparison purposes, and for the same data, Hagoort's $Z_{\mathrm{tp}}$ MBE (Eq. 3) and Vo's CCE-based $Z_{\mathrm{tp}}$ MBE (Eq. 7) analysis (not displayed here) yield similar $\mathrm{G}_{\mathrm{i}}$ estimations of 65.3 BSCF and 65.5 BSCF, respectively. Field Case $\mathrm{B}$ is a gas condensate reservoir located in East Texas reported by Allen and Roe (1950). Reservoir pressure dropped below dew point from the beginning of production. This gas reservoir underwent volumetric depletion throughout its entire production life and was nearly depleted by the end of the reported production history shown in Fig. 12. CVD data for this intermediate gas 


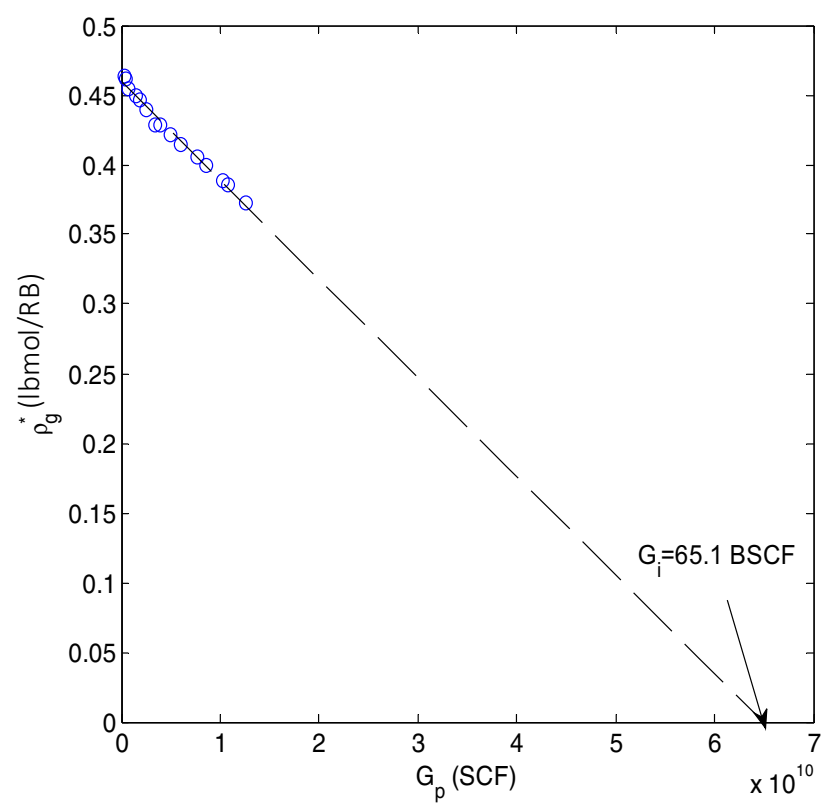

Fig. 11 Density-based material-balance analysis-Field case A

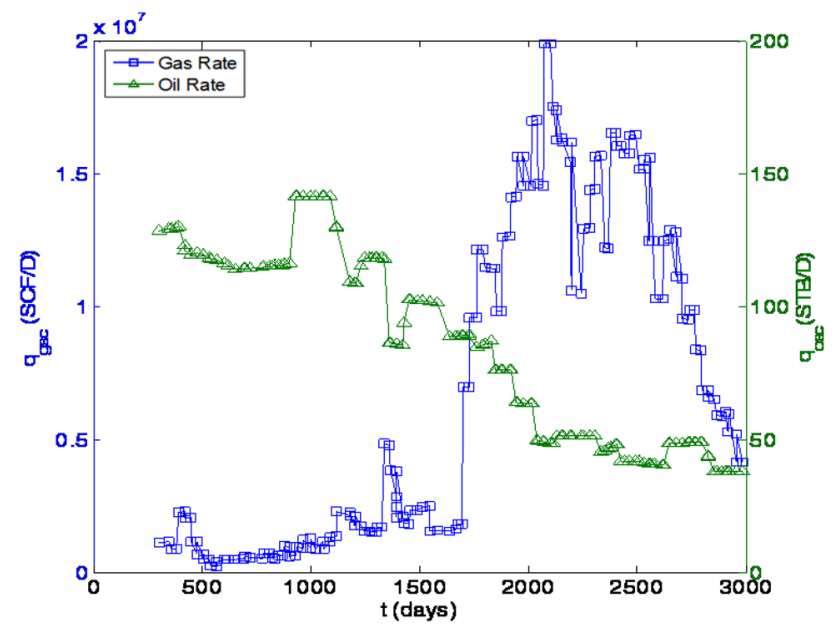

Fig. 12 Production history-Field case B

condensate retrograde shows a $15 \%$ maximum liquid saturation dropout. Figure 13 displays the analysis result using the density-based MBE (Eq. 14), from where original gas and condensate resource are calculated to be 23.0 BSCF and 1.4 MMSTB - consistent with ultimate recovery values of $21 \mathrm{BSCF}$ gas and $0.85 \mathrm{MMSTB}$ condensate at an average pressure of 500 psia reported by the authors. For the same data, Hagoort's $Z_{\mathrm{tp}}$ MBE (Eq. 3) and Vo's CCEbased $Z_{\mathrm{tp}}$ MBE (Eq. 7) analysis (not displayed here) yield similar $G_{i}$ estimations of 23.0 and $22.3 \mathrm{BSCF}$, respectively.

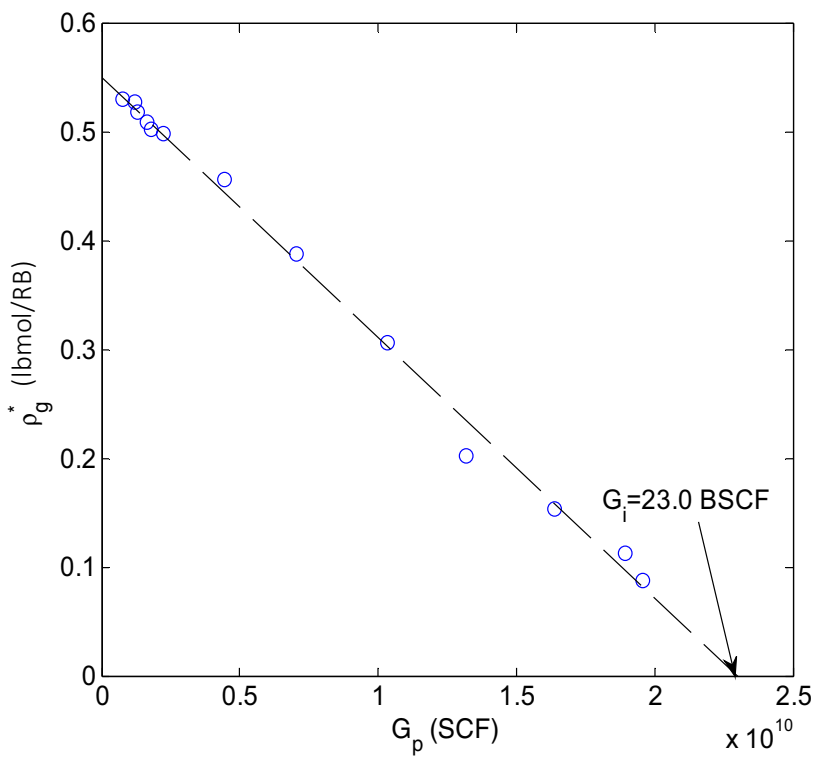

Fig. 13 Density-based material-balance analysis-Field case B

\section{Concluding remarks}

We have reviewed and summarized all available zerodimensional material balance models applicable to liquid-rich gas reservoirs and compared their assumptions. Widely used models-GMBE (Eq. 1) and CVD-based two-phase Z-factor (Eq. 3) - are shown to be consistent with overall molar hydrocarbon conservation and associated black-oil PDEs. It is shown why the application of a simplified two-phase $Z$-factor model (Eq. 7) requires the formulation of a fictitious constant-composition producing stream and CCE-based $Z_{\mathrm{tp}}$ data. A densitybased MBE (Eq. 14) is proposed on the basis of species (surface gas component) conservation. The proposed MBE is written in term of equivalent gas molar density and cumulative surface gas production, and thus can be visualized as a direct extension of the gas density MBE applicable to dry gas reservoirs. The density-based MBE is shown to be easy to implement, to be readily related to CVD test parameters, and to provide reliable average pressure predictions and reserve estimation for a number of numerical and field case studies. The methodology is consistent with other available modified two-phase Zfactor approaches (Eq. 8), but does not require the definition of two-phase $Z$-factors and more closely connects to physical quantities (density) and physical principles, directly tracking the evolution of the surface gas-component storage term on the right-hand-side of the surface-gas PDE. 
Acknowledgments The authors would like to express our gratitude to the members of the Unconventional Natural Resources Consortium (UNRC) at Penn State U. and Foundation CMG (FCMG) for their support of our work in the area of unconventional phenomena analysis in liquid-rich gas plays. The first version of this manuscript was presented as a conference paper at the 2015 SPE Annual Technical Conference and Exhibition as SPE 175081 and the feedback obtained from colleagues in this forum is greatly appreciated.

Open Access This article is distributed under the terms of the Creative Commons Attribution 4.0 International License (http:// creativecommons.org/licenses/by/4.0/), which permits unrestricted use, distribution, and reproduction in any medium, provided you give appropriate credit to the original author(s) and the source, provide a link to the Creative Commons license, and indicate if changes were made.

\section{Appendix 1: Relationship between generalized PDEs and GMBE}

Integrating the surface gas component PDE in Eq. 17 over the entire reservoir volume $V$ yields:

$$
\begin{aligned}
& -\iiint_{V} \nabla \cdot\left(\frac{\mathbf{v}_{V}}{B_{V}}+\frac{\mathbf{v}_{L}}{B_{L}} R_{s}\right) \mathrm{d} V \\
& \quad=-\iiint_{V} \nabla \frac{\partial}{\partial t}\left[\phi\left(\frac{S_{V}}{B_{V}}+R_{s} \frac{S_{L}}{B_{L}}\right)\right] \mathrm{d} V
\end{aligned}
$$

Following Gauss divergence theorem, the volume integral of the divergence over the region inside the surface equals the outward flux of the vector through the closed surface. Therefore, the left-hand-side of Eq. 19 yields total surface gas production out of the control volume:

$$
\begin{gathered}
-\iiint_{V} \nabla \cdot\left(\frac{\mathbf{v}_{V}}{B_{V}}+\frac{\mathbf{v}_{L}}{B_{L}} R_{S}\right) \mathrm{d} V=-\iint_{S}\left(\frac{\mathbf{v}_{V}}{B_{V}}+\frac{\mathbf{v}_{\boldsymbol{L}}}{B_{L}} R_{S}\right) \\
\cdot \widehat{\boldsymbol{n}} \mathrm{d} S=-q_{g s c}
\end{gathered}
$$

Application of Leibniz's rule to the right-hand-side of Eq. 19 yields:

$$
\begin{aligned}
& \iiint_{V} \frac{\partial}{\partial t}\left[\phi\left(\frac{S_{V}}{B_{V}}+R_{s} \frac{S_{L}}{B_{L}}\right)\right] \mathrm{d} V \\
& =\frac{d}{\mathrm{~d} t} \iiint_{V}\left[\phi\left(\frac{S_{V}}{B_{V}}+R_{s} \frac{S_{L}}{B_{L}}\right)\right] \mathrm{d} V=V_{b} \frac{d}{\mathrm{~d} t} \overline{\left[\phi\left(\frac{S_{V}}{B_{V}}+R_{s} \frac{S_{L}}{B_{L}}\right)\right]}
\end{aligned}
$$

Thus, Equation 19 becomes:

$$
-q_{g s c}=V_{b} \frac{d}{\mathrm{~d} t} \overline{\left[\phi\left(\frac{S_{V}}{B_{V}}+R_{s} \frac{S_{L}}{B_{L}}\right)\right]}
$$

where volumetric average is applied for $\phi\left(\frac{S_{V}}{B_{V}}+R_{S} \frac{S_{L}}{B_{L}}\right)$ term. Since tank models assume average value representing entire reservoir condition, all of these parameters $\left(B_{L}, B_{V}\right.$, $R_{s}, R_{v}, S_{L}$ and $S_{V}$ ) stated in this study imply average condition and "-" is dropped subsequently. Further integration of Eq. 22 allows eliminating the timedimension:

$-\int_{0}^{t} q_{g s c} \mathrm{~d} t=V_{b} \int_{0}^{t}\left[\phi\left(\frac{S_{V}}{B_{V}}+R_{s} \frac{S_{L}}{B_{L}}\right)\right] \mathrm{d} t$

which leads to:

$$
\begin{aligned}
-G_{p} & =V_{b}\left[\phi\left(\frac{S_{V}}{B_{V}}+R_{s} \frac{S_{L}}{B_{L}}\right)\right]_{t}-V_{b}\left[\phi\left(\frac{S_{V}}{B_{V}}+R_{s} \frac{S_{L}}{B_{L}}\right)\right]_{t=0} \\
& =V_{b}\left[\phi\left(\frac{S_{V}}{B_{V}}+R_{s} \frac{S_{L}}{B_{L}}\right)\right]-G_{i}
\end{aligned}
$$

Thus,

$\left(-G_{p}+G_{i}\right)=V_{b} \phi\left(\frac{S_{V}}{B_{V}}+R_{s} \frac{S_{L}}{B_{L}}\right)$

Following the same procedure for the surface-oil flow equation, Eq. 18 can also be integrated over the entire reservoir volume to yield:

$\int_{0}^{t} q_{o s c} \mathrm{~d} t=V_{b} \int_{0}^{t}\left[\phi\left(\frac{S_{L}}{B_{L}}+R_{v} \frac{S_{V}}{B_{V}}\right)\right] \mathrm{d} t$

which leads to the following final expression in terms of cumulative stock-tank oil production:

$\left(-N_{p}+N_{i}\right)=V_{b} \phi\left(\frac{S_{L}}{B_{L}}+R_{v} \frac{S_{V}}{B_{V}}\right)$

In this development, in situ water and water production are neglected but could be included without change in the methodology. Equations 25 and 27 can be written for initial condition as:

$G_{i}=V_{b} \phi_{i}\left(\frac{S_{V i}}{B_{V i}}+R_{s i} \frac{S_{L i}}{B_{L i}}\right)$

$N_{i}=V_{b} \phi_{i}\left(\frac{S_{L i}}{B_{L i}}+R_{v i} \frac{S_{V i}}{B_{V i}}\right)$

Removing $S_{L}$ in Eqs. 25 and $27\left(S_{V}=1-S_{L}\right)$ leads to:

$V_{b} \phi=\frac{\left(G_{i}-G_{p}\right)\left(B_{V}-B_{L} R_{v}\right)+\left(N_{i}-N_{p}\right)\left(B_{L}-B_{V} R_{s}\right)}{\left(1-R_{S} R_{v}\right)}$

Removing $S_{L i}$ in Eqs. 28 and $29\left(S_{V i}=1-S_{L i}\right)$ yields:

$V_{b} \phi_{i}=\frac{G_{i}\left(B_{V i}-B_{L i} R_{v i}\right)+N_{i}\left(B_{L i}-B_{V i} R_{s i}\right)}{\left(1-R_{s i} R_{v i}\right)}$

If rock compressibility is assumed to be negligible $\left(\phi_{i} \cong \phi\right)$, Eqs. 30 and 31 lead to: 

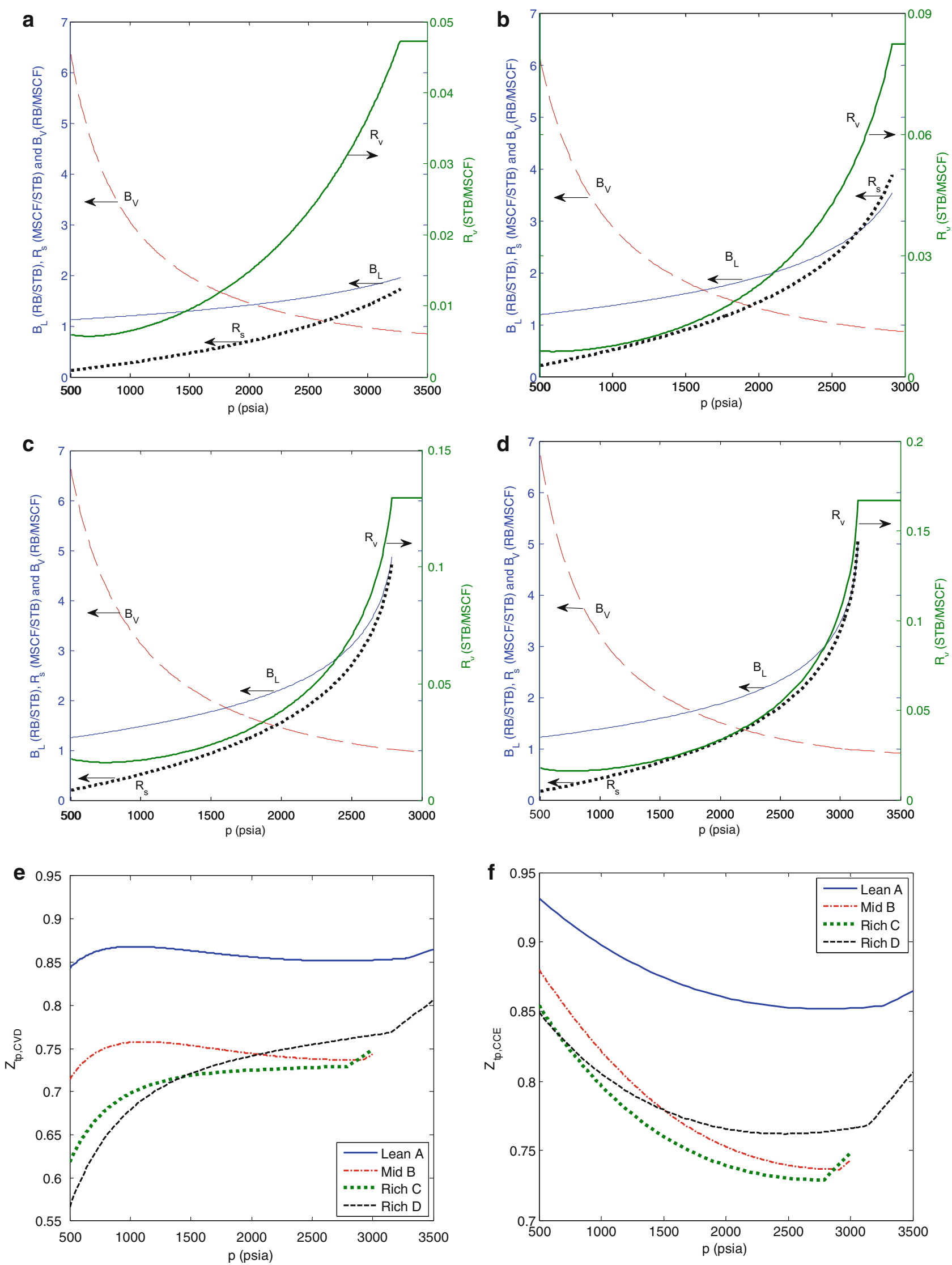
4Fig. 14 a Fluid properties of Lean gas A. b Fluid properties of Mid gas B. c Fluid properties of Rich gas C. d Fluid properties of Rich gas D. e CVD two-phase $Z$-factors for fluids A, B, C, and D. f CCE twophase $Z$-factors for fluids A, B, C, and D

$$
\begin{gathered}
\frac{\left(G_{i}-G_{p}\right)\left(B_{V}-B_{L} R_{v}\right)+\left(N_{i}-N_{p}\right)\left(B_{L}-B_{V} R_{s}\right)}{\left(1-R_{s} R_{v}\right)} \\
=\frac{G_{i}\left(B_{V i}-B_{L i} R_{v i}\right)+N_{i}\left(B_{L i}-B_{V i} R_{s i}\right)}{\left(1-R_{s i} R_{v i}\right)}
\end{gathered}
$$

For an initial reservoir pressure equal or above dewpoint pressure:

$V_{b} \phi_{i}=B_{V i} G_{i}$

$N_{i}=R_{v i} G_{i}$

Substitution of Eqs. 33 and 34 in 30 yields:

$B_{V i} G_{i}=\frac{\left(G_{i}-G_{p}\right)\left(B_{V}-B_{L} R_{v}\right)+\left(R_{v i} G_{i}-N_{p}\right)\left(B_{L}-B_{V} R_{s}\right)}{\left(1-R_{s} R_{v}\right)}$

Or,

$$
\begin{aligned}
& N_{p}\left(B_{L}-B_{V} R_{s}\right)+G_{p}\left(B_{V}-B_{L} R_{v}\right) \\
& \quad=G_{i}\left[R_{v i}\left(B_{L}-B_{V} R_{s}\right)-B_{V i}\left(1-R_{s} R_{v}\right)+\left(B_{V}-B_{L} R_{v}\right)\right]
\end{aligned}
$$

To relate Eq. 32 to the form proposed by Walsh et al. (1994a), we write:

$$
\begin{aligned}
N_{i} & =G_{f g i} R_{v i}+N_{f o i} \\
G_{i} & =G_{f g i}+N_{f o i} R_{s i}
\end{aligned}
$$

where $G_{f g i}$ is initial surface gas present in reservoir vapor phase, and $N_{f o i}$ is initial surface oil present in reservoir liquid phase. Substitution of Eqs. 37 in 32 results in:

$$
\begin{aligned}
N_{p} & \frac{B_{L}\left(1-B_{V} R_{p s}\right)+B_{V}\left(R_{p s}-R_{s}\right)}{\left(1-R_{s} R_{v}\right)} \\
= & G_{f g i} \frac{\left(B_{V}-B_{V i}\right)+B_{L}\left(R_{v i}-R_{v}\right)+R_{s}\left(B_{V i} R_{v}-B_{V} R_{v i}\right)}{\left(1-R_{s} R_{v}\right)} \\
& +N_{f o i} \frac{\left(B_{L}-B_{L i}\right)+B_{V}\left(R_{s i}-R_{s}\right)+R_{v}\left(B_{L i} R_{s}-B_{L} R_{s i}\right)}{\left(1-R_{s} R_{v}\right)}
\end{aligned}
$$

Equation 38 is identical Walsh et al. (1994a)'s GMBE written in terms of fluid withdrawal and expansion. For an initially undersaturated gas condensate reservoir $\left(p_{i}>p_{\mathrm{dew}}\right)$, $N_{f o i}=0$ and $G_{i}=G_{f g i}$; thus Eq. 38 collapse to Eq. 1 as:

$$
\begin{aligned}
& N_{p} \frac{B_{L}\left(1-B_{V} R_{p s}\right)+B_{V}\left(R_{p s}-R_{s}\right)}{\left(1-R_{s} R_{v}\right)} \\
&=G_{i} \frac{\left(B_{V}-B_{V i}\right)+B_{L}\left(R_{v i}-R_{v}\right)+R_{s}\left(B_{V i} R_{v}-B_{V} R_{v i}\right)}{\left(1-R_{s} R_{v}\right)}
\end{aligned}
$$

And it is the same as Eq. 36 after substituting $R_{p s}=$ $G_{p} / N_{p}$.

\section{Appendix 2: Relationship between generalized PDEs and two-phase $Z$-factor MBE}

As shown in Appendix 1, the integration of the surface gas component PDE (Eq. 17) over the entire reservoir volume leads to the total gas flow rate equation in Eq. 22. To derive the twophase- $Z$ MBE first proposed by Hagoort (1988), and thus showcase its consistency with numerical PDEs and GMBE, we express Eq. 22 in terms of molar conservation by multiplying both sides by the gas molar density at standard condition $\left(\rho_{g s c}\right)$ :

$-q_{g s c} \rho_{g s c}=V_{b} \rho_{g s c} \frac{d}{\mathrm{~d} t} \overline{\left[\phi\left(\frac{S_{V}}{B_{V}}+R_{s} \frac{S_{L}}{B_{L}}\right)\right]}$

The integration of Eq. 40 over time yields:

$$
\begin{gathered}
-n_{g p}=\int_{0}^{t}\left(q_{g s c} \rho_{g s c}\right) \mathrm{d} t=V_{b}\left[\phi \rho_{g s c}\left(\frac{S_{V}}{B_{V}}+R_{s} \frac{S_{L}}{B_{L}}\right)\right]_{t} \\
-V_{b}\left[\phi \rho_{g s c}\left(\frac{S_{V}}{B_{V}}+R_{s} \frac{S_{L}}{B_{L}}\right)\right]_{t=0}
\end{gathered}
$$

The first term in the right-hand side of this equation represents the total amount of moles of surface gas left in the reservoir at time ' $t$ ', and the last term represents initial moles of surface gas. Thus, Eq. 41 is just a simple surfacegas molar conservation statement:

$n_{g p}=n_{g i}-n_{g}$

where $n_{g}=n_{g}^{V}+n_{g}^{L}$. Following the same development, Eq. 26 can be multiplied by surface oil molar density $\rho_{\text {osc }}$ to write:

$$
\begin{aligned}
& -n_{o p}=-\int_{0}^{t}\left(q_{o s c} \rho_{o s t}\right) \mathrm{d} t=V_{b}\left[\phi \rho_{\text {ost }}\left(\frac{S_{L}}{B_{L}}+R_{v} \frac{S_{V}}{B_{V}}\right)\right]_{t} \\
& -V_{b}\left[\phi \rho_{\text {ost }}\left(\frac{S_{L}}{B_{L}}+R_{v} \frac{S_{V}}{B_{V}}\right)\right]_{t=0}
\end{aligned}
$$

Similarly, this yields the simple molar statement for surface-oil:

$n_{o p}=n_{o i}-n_{o}$

where $n_{o}=n_{o}^{V}+n_{o}^{L}$. The combination of Eqs. 42 and 44 leads to:

$n_{p}=n-n_{i}$

where $n_{p}$ and $n_{i}$ are produced total moles of hydrocarbon and initial hydrocarbon moles in place, respectively. The definition of the single-phase $Z$-factor concept, applied to both the vapor and liquid reservoir phases, leads to: 
$p V_{V}=n_{V} Z_{V} R T$

$p V_{L}=n_{L} Z_{L} R T$

which, when applied to the overall two-phase system in terms of total volume $\left(V=V_{V}+V_{L}\right)$ and total moles ( $\left.n=n_{V}+n_{L}\right)$, allows writing:

$p V=n Z_{t p} R T$

where $Z_{\mathrm{tp}}$ is the concept of the two-phase $Z$-factor. Addition of Eqs. 46 and 47 and comparison against 48 reveals:

$p\left(V_{L}+V_{V}\right)=\left(n_{V} Z_{V}+n_{L} Z_{L}\right) R T$

from where the observation $n_{V} Z_{V}+n_{L} Z_{L}=n Z_{\text {tp }}$ follows from the inspection of Eqs. 48 and 49. This leads to the identification of the two-phase compressibility factor as a molar-weighted average of single-phase $Z$-factors:

$Z_{\mathrm{tp}}=f_{n V} Z_{V}+f_{n L} Z_{L}$

where $f_{n V}=n_{V} / n$ and $f_{n L}=n_{L} / n$. For the initial condition, we write:

$p_{i} V=n_{i} Z_{t p, i} R T$

Finally, substitution of Eqs. 48 and 51 into Eq. 45, when solved in terms $n$ and $n_{i}$, yields:

$\frac{p}{Z_{\mathrm{tp}}}=\frac{p_{i}}{Z_{\mathrm{tp}, i}}\left(1-\frac{n_{p}}{n_{i}}\right)$

Equation 52 is Hagoort's two-phase Z-factor MBE proposed for gas-condensate reservoirs.

\section{Appendix 3: Fluid CVD and CCE data}

Four different natural gases of varying degrees of initial volatilized condensate contents were considered in this study: Lean gas A, Mid gas B, Rich gas C, and Rich gas D (see Table 1). CVD $B_{L}, B_{V}, R_{s}$ and $R_{v}$ data for these fluids are presented in Fig. $14 \mathrm{a}, \mathrm{b}, \mathrm{c}$, and d, respectively. $Z_{\mathrm{tp}}$ data from CVD and CCE tests for the same fluids is presented in Fig. $14 \mathrm{e}$ and $\mathrm{f}$, respectively.

\section{References}

Allen FH, Roe RP (1950) Performance characteristics of a volumetric condensate reservoir. Pet Trans AIME 189:83-90

Ayala HLF, Ye P (2013) Unified decline type-curve analysis for natural gas wells in boundary-dominated flow. SPE J 18(1):97-113

Bengherbia M, Tiab D (2002) Gas-condensate well performance using compositional simulator: a case study. Paper SPE 75531 presented at the SPE gas technology symposium. Calgary, Alberta, Canada, 30 April-2 May

Hagoort J (1988) Fundamentals of gas reservoir engineering. Elsevier Ltd, Amsterdam

Lee J, Wattenbarger R (1996) Gas reservoir engineering, SPE textbook series, vol 5. Society of Petroleum Engineers, Richardson

Schilthuis RJ (1936) Active oil and reservoir energy. Trans AIME 148:33-52

Sureshjani MH, Clarkson CR, Behmanesh H (2014) A new semianalytical method for analyzing production data from constant flowing pressure wells in gas condensate reservoirs during boundary-dominated flow. Paper SPE 169515 presented at the SPE Western North American and Rocky Mountain Joint Regional Meeting, Denver, Colorado, 16-18 April

Vo DT, Camacho-V RG, Jones JR (1990) A unified treatment of material-balance computations. Paper SPE 21567 presented at SPE international technical meeting, Calgary, Alberta, Canada, 10-13 June

Walsh MP, Lake LW (2003) A generalized approach to primary hydrocarbon recovery. Handbook of petroleum exploration and production, vol 4. Elsevier Ltd, Amsterdam

Walsh MP, Ansah J, Raghavan R (1994a) The new, generalized material balance as an equation of a straight line: part 1applications to undersaturated volumetric reservoirs. Paper SPE 27684 presented at SPE Permian Basin Oil and Gas Recovery Conference held in Midland, Texas, 16-18 March

Walsh MP, Ansah J, Raghavan R (1994b) The new, generalized material balance as an equation of a straight line: part 2applications to saturated and non-volumetric reservoirs. SPE Paper 27728 presented at SPE Permian Basin Oil And Gas Recovery Conference held in Midland, Texas, 16-18 March

Zeidouni M, Movazi GH, Pourghasem B (2006) Performance prediction of a rich gas/condensate reservoir through material balance and PVT Behavior: A case study. Paper SPE 99830 presented at the SPE gas technology symposium. Calgary, Alberta, Canada, 15-17 May

Zhang M, Ayala H LF (2014) Gas-rate forecasting in boundarydominated flow: constant-bottomhole-pressure decline analysis by use of rescaled exponential models. SPE J 19(3):410-417 